\title{
COMPARISON OF FEMTOSECOND ELECTRON BEAM DIAGNOSTIC METHODOLOGIES
}

\author{
J. Sugahara*, T. Watanabe, K. Yoshii, T. Ueda, M. Uesaka \\ Nucl. Eng. Res. Lab., Univ. of Tokyo, 2-22 Shirakata-Shirane, Tokai, Naka, Ibaraki, Japan \\ Y. Kondo \\ School of Eng., Tohoku Univ., Aoba08, Aramaki, Aoba-ku, Sendai, Miyagi, Japan. \\ Y. Shibata, K. Ishi, M. Ikezawa, \\ Res. Inst. Sci. Meas., Tohoku Univ., 2-2-1 Katahira, Aoba-ku, Sendai, Japan.
}

\begin{abstract}
Measurements of longitudinal pulse length of femtosecond electron beams have been performed by the three methodologies at the $35 \mathrm{MeV}$ S-band twin liner accelerators at Nuclear Engineering Research Laboratory, University of Tokyo [1]. The methods we adopt are the femtosecond streak camera with a dispersionless reflective optics, the coherent transition radiation (CTR) Michelson interferometer [2] and the CTR polychromator. The results were compared with one another, and the reliabilities of the methods to diagnose femtosecond electron pulses have been discussed.
\end{abstract}

\section{INTRODUCTION}

Now it has become possible to generate femtosecond electron pulses, which are available for the ultrashort Xray generation and the subpicosecond pulseradiolysis in our facility. In near future, we aim to product and measure a 10 fs (FWHM) electron pulse which pulse length is shorter than the time resolution of the femtosecond streak camera (200 fs at FWHM). Therefore it is necessary to construct an alternative diagnostic system for the femtosecond electron pulse. As the alternative, there are two promising methods to evaluate the subpicosecond pulse shape, both of which use coherent transition radiation (CTR) emitted by the electron pulses in frequency domain. The first is the CTR Michelson interferometer, which utilizes an autocorrelation to obtain a CTR spectrum. The second is the CTR polychromator, which enables us to get a CTR spectrum directly by a single shot. It is very important to compare the results by these methods with that by the femtosecond streak camera in order to confirm the precision of the methods. In this paper, we describe the principle of pulse diagnostics by the methods and measure subpico- and picosecond electron pulses, which are longer than the time resolution of the streak camera.

*Email: jun@ tokai.t.u-tokyo.ac.jp

\section{DIAGNOSTICS BY CTR METHODS}

\section{$2.1 C T R$}

Transition radiation is emitted when an electron passes the interface of two mediums of different dielectric constants. In case that the wavelength of the radiation is longer than the bunch length, the radiation becomes CTR. CTR emitted by electron pulses carries the information of bunch distribution and we can derive the longitudinal shapes of the electron bunch by analyzing the frequency information.

\subsection{Michelson interferometer}

From the interferogram, the power spectrum of the radiation $|E(v)|^{2}$ is given by the Fourier transformation as follows,

$|\tilde{E}(v)|^{2}=\frac{1}{4 \pi c|R T|^{2}} \int_{-\infty}^{+\infty} S(\delta) e^{-i 2 \pi v \delta / c} d \delta$,

where $v$ is the wavenumber, $S(\delta)$ is the light intensity of the recombined radiation at the detector which expressed in the time domain with an additional time delay $\delta / c$ for the movable mirror minus the intensity at $\delta \rightarrow \pm \infty$ and $\mathrm{R}, \mathrm{T}$ are the coefficients of reflection and transmission at the beam splitter, respectively. The longitudinal bunch form factor can be obtained by,

$$
f(v)=\frac{\int_{-\infty}^{+\infty} S(\delta) e^{-i 2 \pi \delta v / c} d v}{4 \pi c|R T|^{2} N^{2} I_{e}(v)}
$$

where $N$ is the number of electrons in the bunch and $\mathrm{I}_{\mathrm{e}}(v)$ is the radiation intensity emitted from a single electron. The longitudinal bunch distributions can be deduced under an assumption of the asymmetric bunch distribution and then the Kramers-Kronig relation is used with the inverse Fourier transformation as follows,

$$
\begin{aligned}
& h(z)=\int_{-\infty}^{+\infty} f_{L}(v) \exp \left[i\left(\phi_{g}(v)-2 \pi v t\right)\right], \\
& \phi_{g}(v)=-2 v \int_{0}^{+\infty} \frac{\ln \left[g\left(v^{\prime}\right)-g(v)\right]}{v^{\prime 2}-v^{2}} d v .
\end{aligned}
$$

Furthermore, we must choose theoretical distribution 
functions of the electron bunch such as a Gaussian distribution or an exponential distribution. The results of these methods and the discussion appear in the following chapter.

\subsection{Polychromator}

Using the polychromator, we can get the spectrum of the radiation directly. From the spectrum, the bunch distribution can be deduced by the same procedure as that by the interferometry. This simplification of the analysis is one of the advantages of the polychromator method. Another advantage is that it enables us to diagnose the electron beam by a single shot. However, the information is very limited by the number of detectors $(10 \mathrm{ch})$. Hence we must be careful in choosing theoretical extrapolation in the procedure of the reconstruction.

\section{EXPERIMENT}

\subsection{Experimental setup}

We performed this comparison at the $35 \mathrm{~L}$ linac where the achromatic-arc-type magnetic pulse compressor was installed. In the experiment the longitudinal bunch distributions were controlled by tuning the energy modulation of the bunch in the accelerating tube for the magnetic pulse compression. We chose subpico- and picosecond pulse widths and performed the comparison among the femtosecond streak camera, the Michelson interferometry and the polychromator measurement as shown in Fig. 1. We measured the transition radiation in the far-infrared region emitted by an electron bunch at the Al-foil put in the air after the $50 \mu \mathrm{m}$-thick Ti window at the end of the 35L linac. We used liquid-He-cooled $\mathrm{Si}$ bolometer as a detector for the far-infrared radiation. The major beam parameters are as follows: the energy was $34 \mathrm{MeV}$, the pulse length is from about 600 fs to 8.0 ps (FWHM) and the electron charge per bunch is controlled to be 10 to $100 \mathrm{pC}$ avoiding the over-scale of the detectors.

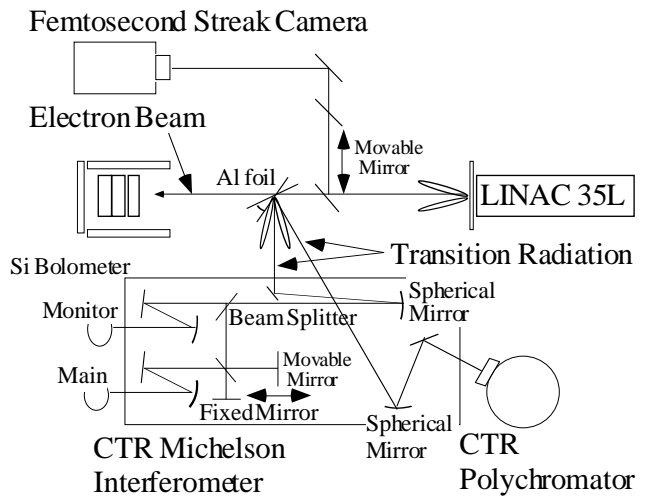

Fig.1 Experimental setup

\subsection{Procedure of analysis}

On the bases of the procedure of analysis in $\mathrm{Ch} 2$, the longitudinal distribution is evaluated. The longitudinal bunch form factors obtained by the two methods were rather limited because of the nonuniform transparency of the $100 \mu \mathrm{m}$-thick Mylar beam splitter in the Michelson interferometer and measurement region which depends on the grating pitch $(1.0 \mathrm{~mm})$ installed in the polychromator. Therefore we have to adopt theoretical extrapolation assuming the Gaussian or exponential distributions out of the range, referring to the pulse shape measured by femtosecond streak camera.

\section{RESULTS AND DISCUSSION}

The CTR spectrum calculated from the interferogram and by the polychromator are shown by the solid curves and the transparency of a $100 \mu \mathrm{m}$-thick Mylar-type beam splitter by dashed curve in Fig.2. From the figure, we decided to use the experimental data in the range of 9.5 to $18.0 \mathrm{~cm}^{-1}$ for the analysis in the interferometry, while the measurable range of the polychromator was already determined from 12.2 to $26.2 \mathrm{~cm}^{-1}$ discretely by the $1 \mathrm{~mm}$ grating pitch.

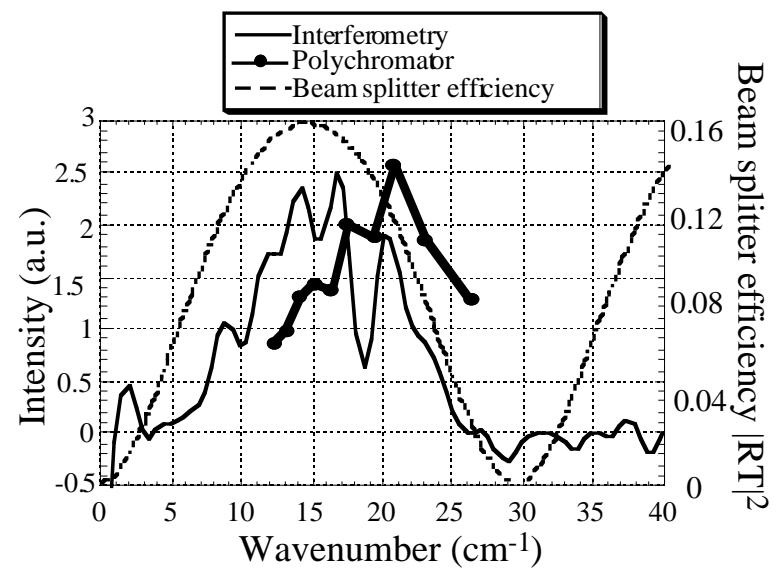

Fig.2. Spectrum of CTR

The experimental results of the longitudinal bunch form factor by the interferometry and by the polychromator are shown by the solid curves and that of theoretical by dashed curve in Fig.3. In the figure, we chose the Gaussian distribution as the theoretical curve, since the exponential distribution has unphysical long tails in both sides. The simultaneous observation of the bunch shapes by the streak camera indicates that the Gaussian distribution is closer to the real bunch distribution. We used the measured bunch form factor in the range that had been described in advance and the theoretical bunch form factor out of the range for the analysis. In the case of the interferometry, we adopt and extrapolate 650 fs (FWHM) bunch length for the subpicosecond pulse and $1.6 \mathrm{ps}$ [FWHM] for the picosecond one, respectively. In the case 
of the polychromator, we chose $1.0 \mathrm{ps}$ for the subpicosecond pulse and 1.4 ps for the picosecond one, respectively.

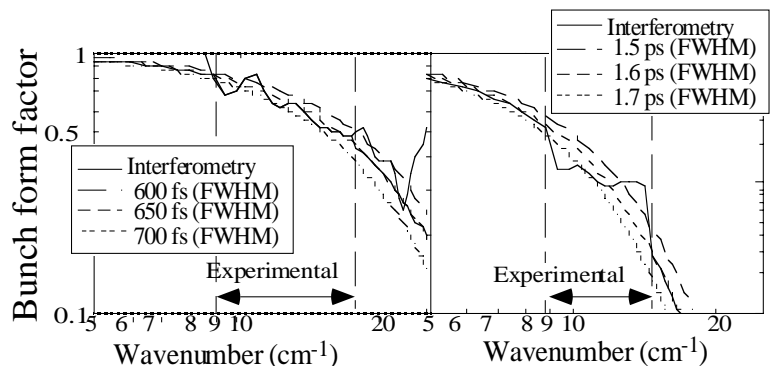

-Results by the interferometry-

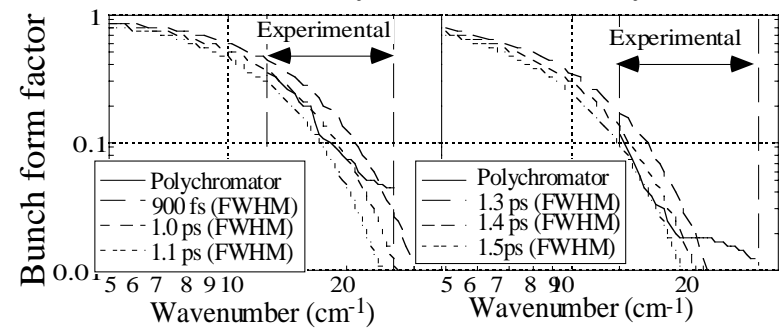

-Results by the polychromator-

Fig.3 Measured longitudinal bunch form factors with the Gaussian fittings

In both cases, the extrapolation for the subpicosecond pulse was more suitable than that for the picosecond one. The reason is that the range of the CTR spectrum measured is more appropriate for the subpicosecond pulse. The longitudinal bunch form factors calculated from the results measured by the streak camera are shown in Fig.4. The important range of the longitudinal bunch form factor to get the coherent effect moves to the larger wavenumber range, as the pulse becomes shorter. We can see that only the bunch form factor of the $1.0 \mathrm{ps}$ or less pulses become smooth in the measurement range, while those of the longer pulses are fluctuated and noisy.

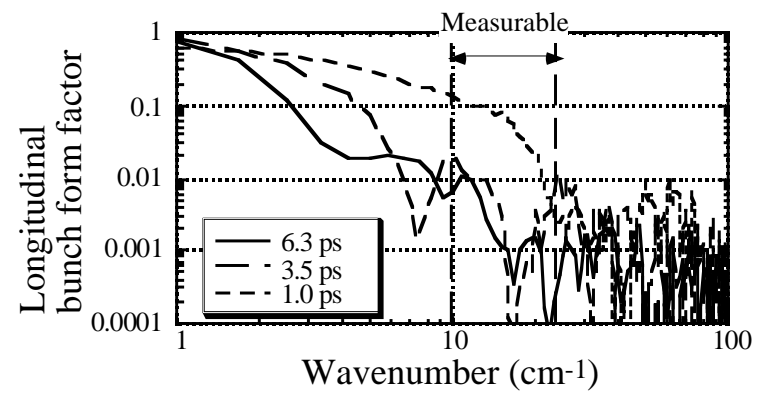

Fig.4 Bunch form factor by the streak camera

Finally, we reconstructed the longitudinal bunch distributions after using Kramers-Kronig relation to derive the phase information. The result of the subpicosecond pulse measurement by the interferometry and that by the polychromator were $650 \mathrm{fs}$ and $1.0 \mathrm{ps}$ at FWHM as shown in Fig.5. Typical result by the streak camera is also shown in the same figure. Here we have got reasonable agreement and confirm the enough reliability of the diagnostics methods by the CTR measurement.

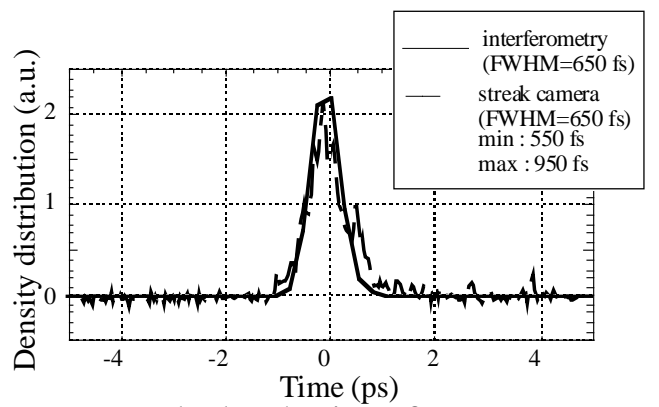

-Results by the interferometry-

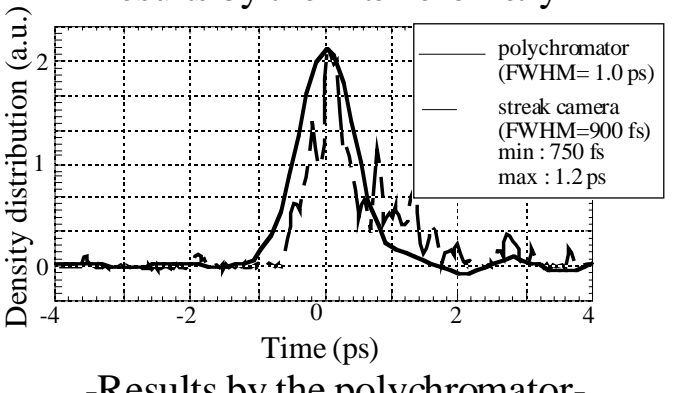

-Results by the polychromator-

Fig.5 Bunch distributions by the three methods

With the choice of a thinner beam splitter or a grating with a narrower pitch, we expect the CTR methods are promising for the shorter electron beam ( $<200 \mathrm{fs}$ ) with better resolution because the spectrum shifts from the farinfrared region to the infrared region where the sensitivity of the detector becomes better. Especially, the polychromator can be expected to the most useful methodology because of the advantage of diagnostics by a single shot.

\section{CONCLUSION}

From the comparison of the results, the reliability of the methods utilizing the CTR to measure subpicosecond electron pulses was confirmed. And we suggested the validity of the femtosecond streak camera for subpicosecond ( $\geq 200 \mathrm{fs}$ ) measurementandpossibility of the polychromator to measure pulses shorter than the resolution of femtosecond streak camera ( $<200 \mathrm{fs})$ in the future.

\section{REFERENCES}

[1] M. Uesaka et al., Nucl. Instr. and Meth. A406 (1998) 371-379.

[2] T. Watanabe, M. Uesaka et al., submitted to Nucl. Instr. and Meth. . 